\title{
Dermatitis por Paederus sp. en la Amazonía peruana: Reporte de
}

\section{casos}

\section{Dermatitis by Paederus sp. in the Peruvian Amazon: Report of cases}

\author{
Martin Fierro-Reinoso ${ }^{1, a}$, Ana Rios-Araujo ${ }^{2, b}$, Erik J. Jhonston ${ }^{3, c}$ \\ ${ }^{1}$ Centro de Salud I3 Santa Clotilde, DIRESA Loreto. Santa Clotilde, Loreto - Perú. \\ ${ }^{2}$ Universidad Nacional de la Amazonia Peruana. Iquitos, Perú. \\ ${ }^{3}$ Unidad de Seguridad y Salud Ocupacional y Medio Ambiente, Hospital Edgardo Rebagliati Martins, Lima - Perú. \\ ${ }^{a}$ Médico-cirujano \\ ${ }^{\mathrm{b}}$ Bióloga \\ ${ }^{\circ}$ Médico Residente de la especialidad de Medicina Ocupacional y Medio Ambiente
}

\section{Correspondencia:}

\section{Erik J. Jhonston}

erik_honston@hotmail.com

Unidad de Seguridad y Salud

Ocupacional y Medio Ambiente,

Hospital Edgardo Rebagliati Martins,

Lima, Perú

Dirección: Avenida Arequipa 4334,

Miraflores. Lima 18, Perú.

Teléfono: (+51) 972838825

Recibido: 15 de septiembre 2017

Aceptado: 15 de marzo 2018

Conflictos de interés: Ninguno.

Fuentes de financiamiento:

Autofinanciado

Contribuciones de autoría: MFR, ARA y EJJ han participado en la concepción del caso reporte, la búsqueda bibliográfica, su redacción y aprobación de la versión final del artículo

Citar como: Fierro-Reinoso M, Rios-Araujo A, Jhonston E. Dermatitis por Paederus sp. en la Amazonía peruana: Reporte de casos. An Fac med. 2018;79(1):49-52 DOI: http://dx.doi.org/10.15381/anales. v79i1.14592
An Fac med. 2018;79(1):49-52 / http://dx.doi.org/10.15381/anales.v79i1.14592

\section{Resumen}

En la amazonia peruana existe una gran diversidad de artrópodos relacionados a problemas de salud pública. Dentro de ellos al poco conocido escarabajo de la familia Staphylinidae, del género Paederus, capaz de producir una dermatitis cáustica vesiculante, a todo aquel que entre en contacto con su hemolinfa. Este tipo de lesiones ha sido descrita en aéreas tropicales y subtropicales causando brotes de dermatitis estacionales a lo largo del mundo, como sucedió en el fenómeno climatológico "El Niño" por Paederus irritans (Ciudad de Piura, 1999). Se presenta dos casos de dermatitis por Paederus sp. en zonas rurales de la amazonia peruana, donde aún no se ha reportado casos.

Palabras clave: Artrópodos; Dermatitis (fuente: DeCS BIREME).

\section{Abstract}

In the peruvian amazon we have a great diversity of arthropods which are related to public health problems. For example, there is an unknown beetle of the family Staphylinidae, genus Paederus, capable of producing a caustic-vesicular dermatitis, to anyone who gets any physical contact with its hemolymph. This type of injury has been described in tropical and subtropical areas, causing epidemic outbreaks of seasonal dermatitis throughout the world; as it happened in the weather phenomenon "El Niño" by Paederus irritans (Ciudad de Piura, 1999). We present two dermatitis cases due to Paederus sp. in rural areas of the peruvian amazon, where no cases have been reported earlier.

Keywords: Arthropods; dermatitis (source: MeSH NLM). 


\section{INTRODUCCIÓN}

El escarabajo del género Paederus (Orden Coleoptera, familia Staphylinidae) está ampliamente distribuido en zonas tropicales y subtropicales del mundo; se conocen 492 especies y al menos el $4 \%$ puede causar dermatitis ${ }^{(1)}$. En el Perú se han identificado 13 especies, de las cuales el Paederus irritans se ha reportado como causante brote de dermatitis, relacionado a el fenómeno “El Niño"(2,3,4). Alrededor del mundo se han descrito más de 70 brotes por Paederus en aproximadamente 40 países $^{(5)}$.

Este artrópodo habita en los campos de cultivo, entre el follaje y hojas se$\operatorname{cas}^{(3,6)}$. De acuerdo a la región geográfica, la población lo identifica con diferentes nombres, por ejemplo: "yuyi" o "zorrito" en Perú (no teniendo un nombre especifico en la amazonia peruana), "bicho del fuego" o "bicho de los vigilantes" en Argentina, "mosca de Nairobi" o "mosca campeón" en Sierra Leona, "araña de drácula" o "balalu" en Irán, "Poto", "fogo-selvagem", "trepa-moleque" y "pélaégua” en Brasil, "Culebrilla” en Colombia, "Cantarida", "Pique-hulle, "Puri-puri" y "Taritari" en Venezuela, "Panchos" en México y en Angola "Ferro em braça" ${ }^{(3,7)}$.

La lesión se caracteriza por una dermatitis (lesiones caustico vesicantes), producida por una sustancia toxica cristalina denominada Paederina, capaz de bloquear la mitosis celular y la síntesis de ADN, provocando eritema, necrosis epidérmica y vesiculación ${ }^{(8,9,10)}$. Los estudios histopatológicos describen cuatro etapas $^{(10)}$ :

- Inicio: primeras 24 a 48 horas caracterizado por edema, eritema, prurito y a veces pápulas.

- Evolución: a partir de las 48 horas se forman vesículas. Entre el quinto y el octavo día, la toxina difunde y da lugar a lesiones secundarias alrededor de la lesión primaria. En esta etapa puede haber dolor, fiebre y malestar general.

- Regresión: a los 8 días disminuye el eritema, se forman costras.

- Regeneración total: se reduce el tamaño de la costra y finalmente cae entre los 20 y los 35 días. No deja cicatriz, sólo una hiperpigmentación residual que posteriormente desaparece.

A continuación, presentamos dos casos de dermatitis por Paederus sp. que se presentaron en localidades rurales del departamento de Loreto.

\section{CASO 1}

Paciente mujer de 26 años de edad, dedicada a la agricultura, sin antecedentes patológicos de importancia; residente de la ciudad de Santa Clotilde (Capital del Distrito del Napo, en la provincia de Maynas en Loreto) acudió al centro de salud en noviembre del año 2015, presentando lesiones dérmicas en ambas piernas de 4 días de evolución, refiriendo que normalmente cuando realiza su labor agrícola, tiene contacto con múltiples artrópodos (que en ocasiones mata por aplastamiento contra la piel), pero no recuerda mordedura o picadura previa a aparición de lesiones. Refiere prurito intenso continuo asociado a eritema linear en las primeras 24 horas, posteriormente la aparición de lesiones vesiculosas dolorosas con trayecto lineal sobre la lesión primaria en las 48 horas siguientes, asociado e escaso dolor quemante en zona afectada.

Al examen físico se aprecian dos lesiones, en la cara medial de pierna derecha e izquierda respectivamente, las lesiones fueron vesiculo pustulosas eritematosas con una disposición lineal de bordes definidos y elevados de $5 \mathrm{~cm}$ y $4 \mathrm{~cm}$ de largo respectivamente (Figura 1).

Los principales diagnósticos diferenciales planteados fueron larva migrans cutánea e infecciones por herpes zóster; la conclusión de dermatitis linear por Paederus se realizó tomando en cuenta la anamnesis, forma de presentación, y por antecedentes previos en la comunidad de que el causante podría ser un artrópodo. Se logró capturar un espécimen en el lugar donde se produjo la exposición, el
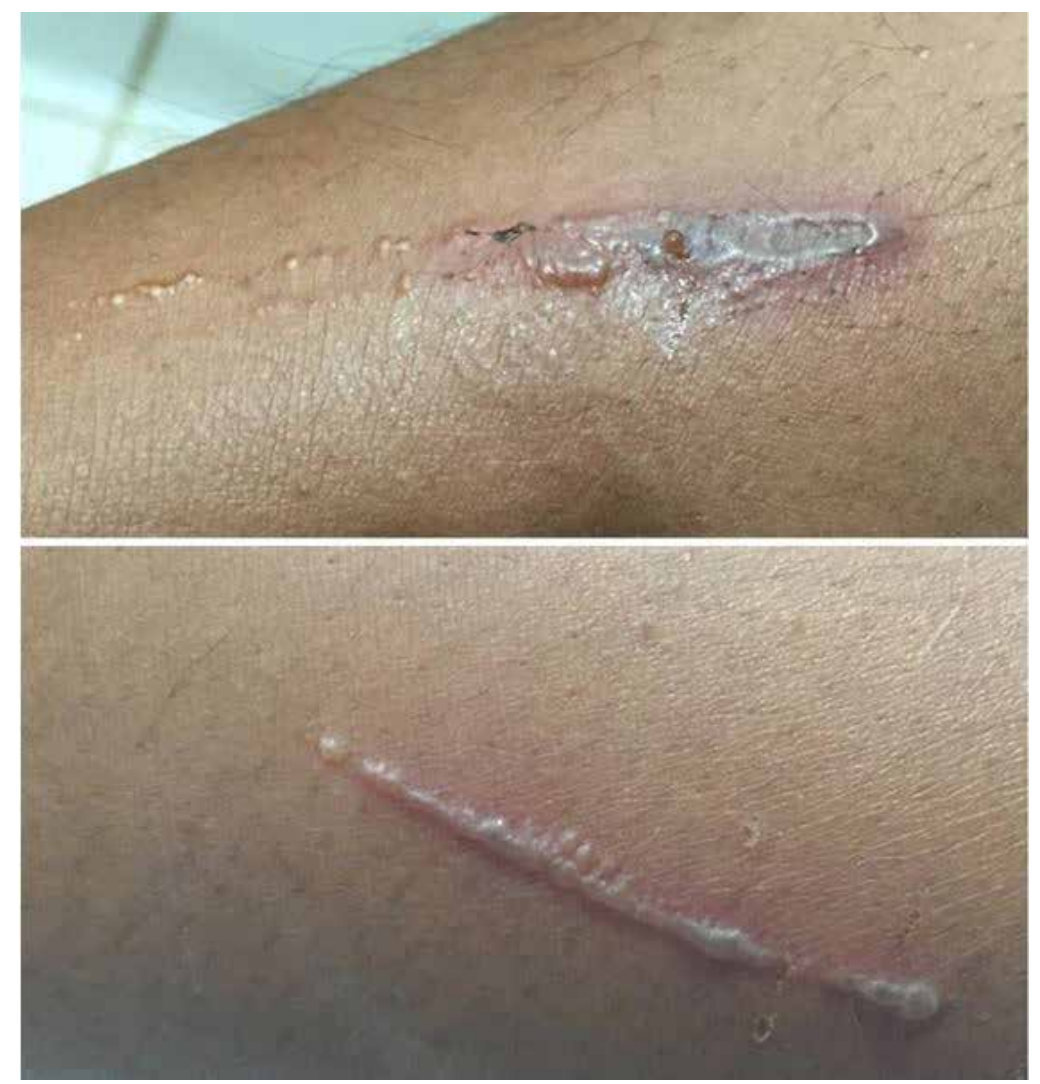

Figura 1. Dermatitis linear por Paederus, pierna derecha e izquierda. Rio Napo, Loreto. 
espécimen se envió a la Facultad de Ciencias Biológicas de la Universidad Nacional de la Amazonia Peruana (UNAP), donde el estudio taxonómico determinó que se trataba de Paederus sp. (Figura 2).

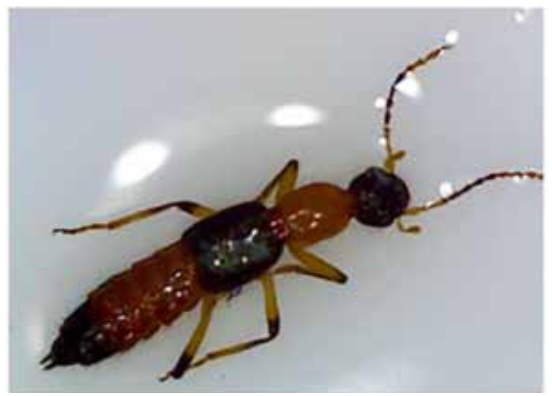

Figura 2. Paederus sp. capturado en el lugar de accidente. Rio Napo, Loreto.

Las lesiones fueron tratadas con corticoides tópicos y antihistamínicos vía oral por 4 dias, con lo cual el cuadro tuvo una evolución favorable y resolución en 4 días posteriores, sin presentar secuelas en la piel afectada.

\section{CASO 2}

Paciente mujer de 25 años, sin antecedentes patológicos, profesional bióloga, residente de la ciudad de lquitos, se encontraba realizando estudios de campo en la cuenca del rio Putumayo, provincia del Putumayo, frontera entre Perú y Colombia, durante noviembre y diciembre del 2016, a bordo de un bote motor de pasajeros durante aproximadamente 10 días, evidenciando en las cercanías de la comunidad peruana de Huapapa la presencia de abundantes insectos del genero Paederus. A los 2 días de evidenciarlos presentó lesiones vesiculares lineares en miembros superiores e inferiores, en el transcurso de 5 días estas se volvían costras para posteriormente desprenderse dejando un lesiones hipocromías que persistieron 45 días después de la lesión primaria (Figura 3), concomitantemente presento conjuntivitis química en el ojo derecho de 4 días de evolución caracterizado por un dolor quemante intenso y eritema de la conjuntiva. La paciente manifestó contacto con Paederus en su camarote, adicionalmente refirió que de las personas a bordo (70 en promedio), 15 presentaron lesiones dérmicas similares a las descritas anteriormente, definición que cumple el criterio de brote, que no pudo ser descrito adecuadamente.

La paciente en su calidad de bióloga decidió recolectar dos especímenes, los cuales llevo a la Facultad de Ciencias Biológicas de la UNAP, donde el estudio taxonómico confirmo que se trataba de Paederus sp. coincidiendo con el espécimen recolectado en Santa Clotilde. Descartándose de que se trate de Paederus irritans por claves taxonómicas.

\section{DISCUSIÓN}

Alrededor del Mundo se ha descrito numerosos brotes de dermatitis causada por escarabajos de la especie Paederus, distribuidos en todo el mundo, excepto en la Antártida; países vecinos como Brasil, Argentina, Ecuador, Panamá, Venezuela también cuentan con reportes de dermatitis por Paederus(1); en el Perú, la dermatitis por Paederus ha sido descrita en un brote ocurrido en la ciudad de Piura, durante el fenómeno "El niño" de 1999, notificándose 1451 casos, la especie involucrada fue el Paederus irritans ${ }^{(3)}$. Lamentablemente debido a los pocos especímenes capturados para este reporte no se logró definir la especie de Paederus causante de la dermatitis, y desde el año 1999 no se ha reportado otros casos por una especie distinta, a pesar de la variedad de especies descritas en nuestro país. Actualmente existe la necesidad de estar alertas a los fenómenos naturales que pueden favorecer el contacto de esta especie con los seres humanos y su potencial daño no solo a la población civil sino también a las bases militares y a la actividad turística ${ }^{(2,11,12,13)}$.

El diagnóstico de la dermatitis por Paederus es clínico, tomando en cuenta la anamnesis, las características de las lesiones y el contexto epidemiológico, tal como se realizó para los casos mencionados $^{(3,9,14)}$. La historia clásica es el contacto con fluidos del escarabajo en la piel, con posterior aparición de lesiones tipo vesículas, asociado a ardor, eritema y edema ${ }^{(3)}$; la disposición lineal de las lesiones constituye una clave para el diagnóstico y se conoce como "signo del latigazo" presentándose en un 57\% de los casos, concordante a los casos sucedidos durante el fenómeno "El Niño"(14). Otras formas de presentación son del tipo "en beso", "herpetiforme", "bizarra" e incluso se han descrito casos no muy frecuentes de conjuntivitis ${ }^{(9)}$, como nuestro segundo caso; la zonas del cuerpo más afectadas

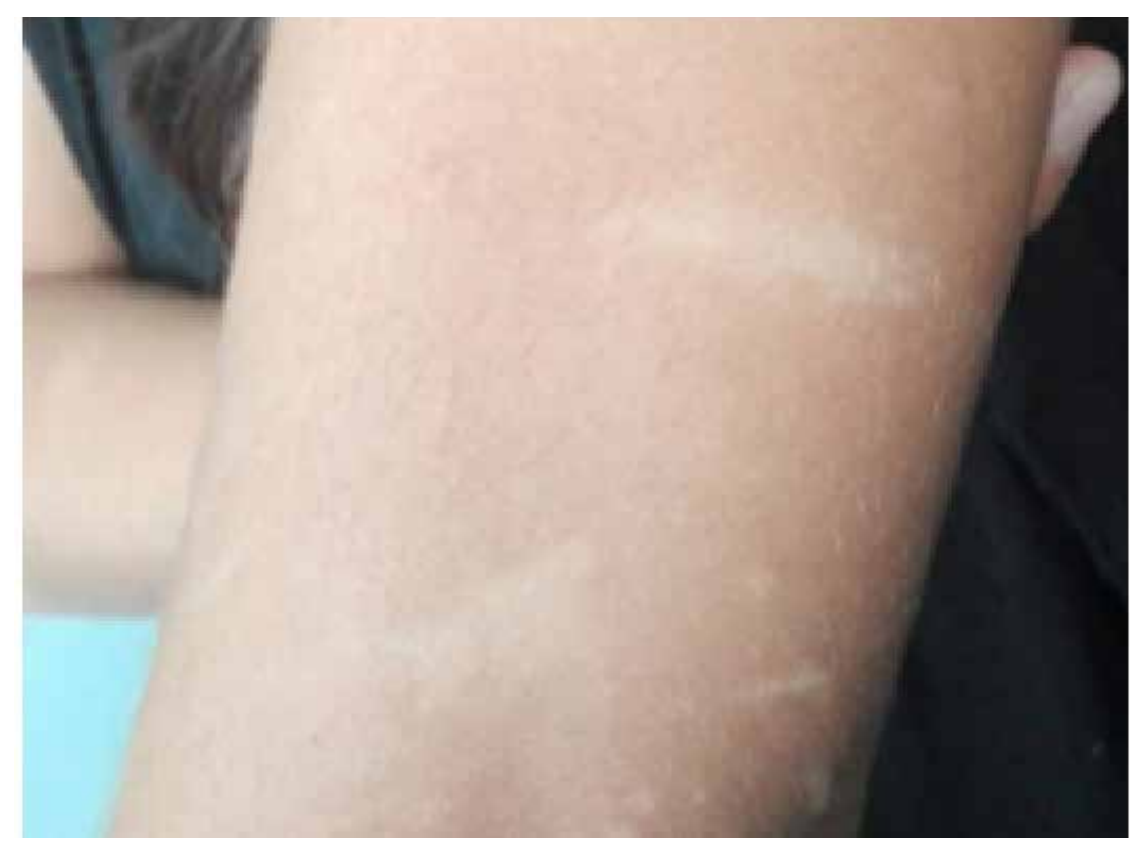

Figura 3. Lesión tardía por Paederus, brazo izquierdo. Rio Putumayo, Loreto 
corresponden a la mitad superior del cuerpo, especialmente en áreas expuestas, principalmente la cara y el cuello, en un $46 \%$ y $26 \%$ respectivamente. Dentro del diagnóstico diferencial que se planteó se encuentra la dermatitis de contacto alérgica o irritativa aguda, quemaduras térmicas, quemaduras químicas, herpes zoster, herpes simple, impétigo ampolloso, fitofotodermatitis y larva migrans cutánea $(7,9,14,15)$.

Los casos presentados son los primeros reportados en la Amazonía peruana, plateando la incógnita del real alcance de esta patología, y su impacto en la salud pública, afectando comunidades de las cuencas mencionadas, bases militares y turismo en la Amazonía (12,13). Asimismo, existe un preocupante subregistro, probablemente debido a su baja morbilidad y nula mortalidad asociado al poco acceso que las comunidades rurales de esta cuenca a los servicios de salud, lo que plantea la necesidad de sensibilizar al personal de salud y de realizar estudios entomológicos en la región para determinar las especies circulantes, su estacionalidad, la interacción que desarrollan con el ser humano y su impacto en la salud de población residente y foránea de las cuencas mencionadas y su posible impacto en situaciones de emergencia.

\section{REFERENCIAS BIBLIOGRÁFICAS}

1. Vieira J, Stramare Ribeiro-Costa C, Caron E. Rove beetles of medical importance in Brazil (Coleoptera, Staphylinidae, Paederinae). Rev Bras Entomol. 2014;58:244-60. DOI: http://dx.doi.org/10.1590/ S0085-56262014000300005

2. Asenjo A. Lista preliminar de las especies de Staphylinidae (Coleóptera) registradas para Perú. 15 de marzo de 2018; Arquivos Entomolóxicos 18: 289-296.

3. Alva-Dávalos $\mathrm{V}$, Laguna-Torres VA, Huamán A, Olivos R, Chávez M, Garcia C, et al. Epidemic dermatits by Paederus irritans in Piura, Perú at 1999, related to El Niño phenomenon. Rev Soc Bras Med Trop. 2002;35(1):23-8. DOI: http://dx.doi. org/10.1590/S0037-86822002000100005

4. Ojeda D. Estudio sobre un Caso de 'Dermatitis producida por Paederus irritans Chapin (col:. Staphilinidae). Rev. Per. de Ent. 1967; 10(1): 28-31.

5. Bong L-J, Neoh K-B, Jaal Z, Lee C-Y. Paederus Outbreaks in Human Settings: A Review of Current Knowledge. J Med Entomol. 2015;52(4):517-26. DOI: 10.1093/jme/tjv041

6. Awad SS, Abdel-Raof H, Hosam-EIDin W, ElDomyati M. Linear neutrophilic dermatitis: A seasonal outbreak of Paederus dermatitis in upper Egypt. Cutis 2013;91:300-4.

7. Garcia Rivas L, Parra JJ, Reyes Flores O. Dermatitis por Paederus en el Estado Cojedes Venezuela. Dermatol Venez. 2001;39(4):93-8.
8. Ahmed M, Boraei H, Rakha O. Histopathological characterization of induced Paederus dermatitis caused by Egyptian rove beetles (Paederus alfierii). Beni suef univercity journal of basic and applied science. 2013; 2: 108-13. DOI: https://doi. org/10.1016/j.bjbas.2013.03.002

9. Mittal A, Mehta S, Garg A, Gupta L, Kuldeep C, et all. A Study of Beetle Dermatitis. Indian Journal of Clinical Practice. 2013; 23(11): 714-16.

10. Guillen Z. Lesiones cutaneas producidas por Paederus irritans (Coleoptera, Staphylinidae) sobre animales de laboratorio. Rev. per. Ent. 1989; 31:31-35.

11. Suarez-Ognio L, Estela-Ayamamani D, CáceresMejía B, Gambirazio-Carbajal C, Cabrera R. Impacto del fenómeno «El Niño» de 1997-1998 en la salud de la población peruana, riesgo potencial para el 2015. Rev Peru Med Exp Salud Publica. 2015;32(2):403-4.

12. Todd RE, Guthridge SL, Montgomery BL. Evacuation of an Aboriginal community in response to an outbreak of blistering dermatitis induced by a beetle (Paederus australis). Med $\mathrm{J}$ Aust.1996;164(4):238-40.

13. Davidson SA, Norton SA, Carder MC, Debboun M. Outbreak of dermatitis linearis caused by Paederus ilsae and Paederus iliensis (Coleoptera: Staphylinidae) at a military base in Iraq. US Army Med Dep J. 2009;6-15.

14. Pagottoa B, Plafnika R, Castilloa A, Cioncia J, Abad $\mathrm{M}$, el all. Dermatitis de contacto por Paederus en un niño de 12 años. Arch Argent Pediatr. 2013; 111(3): e66-e68. DOI: http://dx.doi.org/10.5546/ aap.2013.e66

15. Oborski MR, Capelli L, Montardit AL, Carabajal G, Mazzini MA. Dermatitis por contacto por Paederus. Dermatol Argent. 2015;21(1):56-8. 